\title{
Response to corticosteroid treatment in paediatric nephrotic syndrome: A retrospective review from Mozambique
} \author{
D Puliyanda, ${ }^{3} \mathrm{MD}$ \\ ${ }^{1}$ Hospital Central de Maputo, Maputo, Mozambique \\ ${ }^{2}$ University of California Los Angeles, David Geffen School of Medicine, Los Angeles, California, USA \\ ${ }^{3}$ Cedars-Sinai Medical Center, Los Angeles, California, USA \\ ${ }^{*}$ Co-first author
}

Y Monteiro, ${ }^{1} \mathrm{MD}$; K Faktor, ${ }^{2 *} \mathrm{MSc} ; \mathrm{W}$ C Buck, ${ }^{1,2} \mathrm{MD}$, MSPH; S Taunde, ${ }^{1} \mathrm{MD}$, MPH; E Jeque,${ }^{1} \mathrm{MD} ; \mathrm{S}$ Kakar, ${ }^{1} \mathrm{MD}$;

Corresponding author: W C Buck (wbuck@mednet.ucla.edu)

\begin{abstract}
Background. First-line treatment for paediatric nephrotic syndrome, a chronic disease characterised by proteinuria, hypoalbuminaemia and oedema, is corticosteroid therapy. An association between African ethnicity and steroid resistance has been reported, but published data from sub-Saharan Africa are limited and lack consensus on the prevalence of steroid-resistant disease.

Objective. We aimed to describe a cohort of paediatric patients with nephrotic syndrome, determine the frequency of steroid resistance, and investigate factors associated with steroid resistance at Hospital Central de Maputo, Mozambique.

Methods. A retrospective chart review was performed for paediatric patients ( 1 - 14 years old) admitted with nephrotic syndrome between 2010 and 2015 with at least one follow-up visit by 31 March 2016. Patients with HIV or malaria were excluded. Descriptive statistics and frequencies disaggregated by treatment response were produced. Associations of treatment response with sociodemographic, clinical and laboratory variables were investigated by appropriate univariable statistical tests.

Results. Twenty-eight patients met inclusion criteria and were characterised as having steroid-sensitive or steroid-resistant nephrotic syndrome based on response to an initial corticosteroid regimen. There were 15 boys (53.6\%) and 13 girls (46.4\%), with a median age of 5.5 years. Fifteen $(53.6 \%)$ were steroid-sensitive, and $13(46.4 \%)$ were steroid-resistant. Steroid-resistant patients had significantly lower diastolic blood pressure $(p=0.004)$, lower serum haemoglobin $(p=0.003)$, lower serum albumin $(p=0.03)$ and higher platelet counts ( $p=0.008)$ compared with sensitive cases. Only 4 (30.8\%) steroid-resistant patients received a kidney biopsy.

Conclusion. Given the relatively high occurrence of steroid resistance in this study, expanding access to kidney biopsy and second-line medications for paediatric patients not in remission after an initial course of corticosteroid therapy is needed to meet the current standard of care. Factors associated with steroid resistance need to be studied prospectively in larger cohorts of Mozambican children.
\end{abstract}

S Afr J Child Health 2021;15(1):38-43. https://doi.org/10.7196/SAJCH.2021.v15i1.1780

Nephrotic syndrome is one of the most common causes of kidney disease in paediatric patients, with disadvantaged populations suffering disproportionately from the disease and its complications. ${ }^{[1-3]}$ Data on the clinical profile of paediatric nephrotic syndrome, a chronic condition characterised by proteinuria, hypoalbuminaemia, and oedema, are limited within sub-Saharan Africa (SSA). ${ }^{[4]}$ Consequently, medical management and outcomes of paediatric patients with nephrotic syndrome across SSA are not well established, especially for cases of steroidresistant disease.

Early age at onset is associated with an increased prevalence of steroid-resistant nephrotic syndrome (SRNS) in children. Based on information collected from the PodoNet Consortium international registry including 1655 paediatric patients, childhood-onset SRNS manifested before the age of 5 in $64 \%$ of patients. ${ }^{[5]}$ Historically, more than $50 \%$ of these patients with steroid resistance had progressed to chronic kidney disease (CKD) and end-stage renal disease (ESRD) within five years, particularly within resourcelimited settings. ${ }^{[6]}$ Until the 1980s, quartan malaria nephropathy was prevalent in tropical regions of Africa, making corticosteroidresistant disease common throughout the region. ${ }^{[4]}$ However, given improving access to healthcare for the treatment of malaria, nephrotic syndrome is now increasingly associated with other disease processes, including hepatitis B, HIV, sickle cell disease and systemic lupus erythematosus. ${ }^{[7-9]}$

Response to steroid therapy has also been reported to differ according to geography. ${ }^{[6,10]}$ Studies concerning associations of SRNS with ethnicity present varying conclusions. Retrospective chart reviews reported higher rates of SRNS at institutions in the Democratic Republic of the Congo and Nigeria than those reported in Asian or Western countries (around 10\%). ${ }^{[11-14]}$ Conversely, two recent studies in Nigeria showed improved steroid-sensitivity in cohorts of children. ${ }^{[15,16]}$ Moreover, studies from Zimbabwe, Malawi and South Africa show a continued predominance of focalsegmental glomerulosclerosis (FSGS) and membranoproliferative glomerulonephritis (MPGN) in the region, a histopathologic diagnosis commonly associated with the steroid-resistant disease ${ }^{[17-19]}$ Considering the lack of a definitive association with SRNS within current literature, further characterisation of the clinical profile of SRNS is needed.

The present study aims to address this gap by characterising the baseline clinical and demographic factors associated with steroid resistance in a cohort of Mozambican children with nephrotic syndrome. 


\section{Methods \\ Study design and setting}

This was a retrospective cohort study of children with nephrotic syndrome conducted at Hospital Central de Maputo (HCM), Mozambique's largest referral hospital and the largest teaching hospital in the country. The Department of Paediatrics at HCM has a nephrology service with two paediatric nephrologists. HCM does have peritoneal and haemodialysis available, but children have limited access. Paediatric care is also constrained by limited laboratory and pathology capacity for renal disease diagnosis.

\section{Study participants}

Paediatric patients with nephrotic syndrome who were either active in care or who had previously received care with the paediatric nephrology service at HCM were identified through a review of inpatient and outpatient registers. All children from 1 - 14 years of age at initial presentation, seen between 1 January 2010 and 31 December 2015, with at least one follow-up visit up to 31 March 2016 and an initial course of treatment with corticosteroids were eligible for inclusion in the study. Patients less than one year of age, who probably had a congenital form of nephrotic syndrome, as well as patients who were positive for HIV or malaria on admission, were excluded, given the potential differences in aetiology. ${ }^{[20]}$

\section{Data collection}

Patient charts were retrieved from the hospital archives and reviewed by members of the study team. Demographic and clinical data were extracted from the charts by study investigators onto paper data collection forms which were later entered into a Microsoft Excel (Microsoft Corp., USA) database.

\section{Definition of terms}

In accordance with the hospital's corticosteroid protocol, patients initially received $60 \mathrm{mg} / \mathrm{m}^{2} /$ day or $2 \mathrm{mg} / \mathrm{kg} /$ day of prednisolone with tapering initiated at $6-8$ weeks. Response to corticosteroid therapy was defined using Kidney Disease Improving Global Outcomes $(\mathrm{KDIGO})^{[21]}$ standards which were adapted, given the availability of data for our cohort:

1. Steroid-sensitive nephrotic syndrome (SSNS): Complete remission, $\leq 1+$ proteinuria at the time point closest to, but not exceeding, 12 weeks (was not possible to confirm 3 consecutive days of $\leq 1+$ proteinuria).

2. Steroid-resistant nephrotic syndrome (SRNS): $>2+$ proteinuria and/or oedema at the time point closest to, but not exceeding, 12 weeks.

Hypertension was defined as systolic or diastolic blood pressure greater than the 95th percentile for age and sex, as designated in the Fourth Report of the National High Blood Pressure Education Program. ${ }^{[22]}$ The 50th percentile was used for height, as this information was not routinely available in hospital charts. All measurements were taken manually with an appropriate cuff size. Owing to inconsistent documentation in patient charts, blood pressure measurements were based only on the initial blood pressure measurement recorded at the time of admission.

\section{Data analysis}

Descriptive statistics were produced reporting means for normally distributed continuous variables and medians for abnormally distributed continuous variables. Relative frequencies were determined for categorical variables disaggregated by treatment response. Categories of continuous laboratory variables were produced using normal reference ranges. For the two laboratory variables where all patients had abnormal results (serum albumin and cholesterol), the categories were formed by choosing a cut-off value which resulted in groups of approximately the same size. For analysis of factors associated with treatment response, Fisher's exact test was used for independent categorical variables, $t$-test for continuous normally distributed variables and the WilcoxonMann-Whitney test for continuous variables not normally distributed. Normal distribution of the variables was determined by the Shapiro-Wilk test. All analyses were performed with Stata software.

\section{Ethical considerations}

The protocol was approved by the HCM Scientific Directorate, the University of California Los Angeles Institutional Review Board (ref. no. IRB\#17-000154) and the Institutional Review Board of Universidade Eduardo Mondlane/Hospital Central de Maputo (ref. no. CIBS FM\&HCM/22/2016). Informed consent from patients or caregivers was not required.

\section{Results}

There were 39 patients with nephrotic syndrome identified from the registers during the time period of study eligibility. Charts were successfully located for 34 , of whom 28 patients met inclusion criteria after review. Of the excluded patients, two patients received initial corticosteroid treatment prior to transfer to HCM, one patient had an incomplete chart with an extensive history of hospitalisations for nephrotic syndrome-related complications prior to the study beginning, one patient was less than one year of age at diagnosis, one patient was HIV-positive, and one patient had malaria on admission. The final cohort included 28 patients; $15(53.6 \%)$ were sensitive to prednisolone treatment and 13 (46.4\%) were resistant (Fig. 1).

The demographic and clinical profile of the cohort as categorical variables is detailed in Table 1 . The study cohort was $53.6 \%$ male

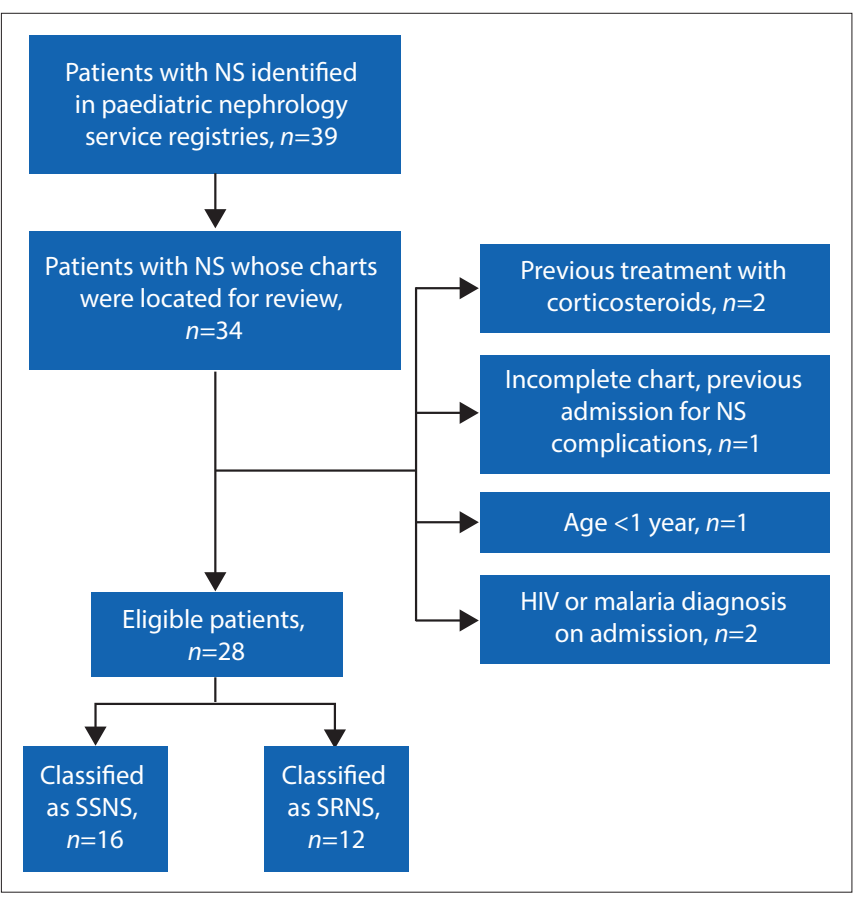

Fig. 1. Study inclusion and steroid response classifications. (NS = nephrotic syndrome; SSNS = steroid-sensitive nephrotic syndrome; SRNS = steroidresistant nephrotic syndrome.) 


\begin{tabular}{|c|c|}
\hline Variable & $N=28, n(\%)$ \\
\hline \multicolumn{2}{|l|}{ Age, years } \\
\hline$<5$ & $12(42.9)$ \\
\hline$>5$ & $16(57.1)$ \\
\hline \multicolumn{2}{|l|}{ Sex } \\
\hline Male & $15(53.6)$ \\
\hline Female & $13(46.4)$ \\
\hline \multicolumn{2}{|l|}{ Oedema } \\
\hline Localised & $1(3.6)$ \\
\hline Generalised & $27(96.4)$ \\
\hline \multicolumn{2}{|c|}{ Blood pressure classification } \\
\hline Normal & $14(50.0)$ \\
\hline High & $12(42.9)$ \\
\hline Missing & $2(7.1)$ \\
\hline \multicolumn{2}{|l|}{ Proteinuria } \\
\hline $1+/ 2+$ & $15(53.6)$ \\
\hline $3+/ 4+$ & $13(46.4)$ \\
\hline \multicolumn{2}{|l|}{ Haematuria } \\
\hline No & $14(50.0)$ \\
\hline Yes & $14(50.0)$ \\
\hline \multicolumn{2}{|c|}{ Serum WBC, $\times 10^{3} / \mathrm{mL}$} \\
\hline$<11$ & $13(46.4)$ \\
\hline$>11$ & $15(53.6)$ \\
\hline \multicolumn{2}{|c|}{ Haemoglobin, g/dL } \\
\hline$>12$ & $6(21.4)$ \\
\hline$<12$ & $22(78.6)$ \\
\hline \multicolumn{2}{|c|}{ Platelets, $\times 1000 / \mathrm{L}$} \\
\hline$<400$ & $7(25.0)$ \\
\hline$>400$ & $21(75.0)$ \\
\hline \multicolumn{2}{|l|}{ Urea, mmol/L } \\
\hline$<7.2$ & $21(75.0)$ \\
\hline$>7.2$ & $7(25.0)$ \\
\hline \multicolumn{2}{|c|}{ Creatinine, $\mu \mathrm{mol} / \mathrm{L}$} \\
\hline$<113$ & $25(89.3)$ \\
\hline$>113$ & $2(7.1)$ \\
\hline Missing & $1(3.6)$ \\
\hline \multicolumn{2}{|l|}{ Albumin, $g / L^{\dagger}$} \\
\hline$<35$ & $28(100)$ \\
\hline$>35$ & $0(0)$ \\
\hline \multicolumn{2}{|c|}{ Cholesterol, $\mathrm{mmol} / \mathrm{L}^{\dagger}$} \\
\hline$<5$ & $0(0)$ \\
\hline$>5$ & $27(96.4)$ \\
\hline Missing & $1(3.6)$ \\
\hline
\end{tabular}

and $42.9 \%$ of patients were $<5$ years of age. Median age was 5.5 years (interquartile range (IQR) 3.7 - 7.9). Regarding ethnicity, there were 27 black patients and one Asian patient. Screening for viral hepatitis was limited, with one patient tested for hepatitis A, five patients tested for hepatitis B, and three patients tested for hepatitis C, all having negative results. Clinically, 27 patients (96.4\%) presented with generalised oedema, and of the 26 patients with documented blood pressure, $12(42.9 \%)$ were classified as hypertensive.

Urinalysis at presentation revealed $3+/ 4+$ proteinuria in 13 patients (46.4\%) and haematuria in 14 patients (50.0\%).

\section{Table 2. Steroid response by categorical variables}

\begin{tabular}{llll} 
Variable & $\begin{array}{c}\text { Steroid- } \\
\text { sensitive, } \\
n(\%)\end{array}$ & $\begin{array}{c}\text { Steroid- } \\
\text { resistant, } \\
\boldsymbol{n}(\%)\end{array}$ & $\boldsymbol{p}$-value* \\
\hline Age & & & \\
$\quad<5$ years & $5(41.7)$ & $7(58.3)$ & 0.445 \\
$>5$ years & $10(62.5)$ & $6(37.5)$ &
\end{tabular}

Sex

Male

Female

$7(46.7) \quad 8(53.3) \quad 0.476$

Blood pressure classification

Normal
High
Missing

Proteinuria

$1+/ 2+$

$3+/ 4+$

Haematuria

No

Yes

$8(61.5) \quad 5(38.5)$

$6(42.9) \quad 8(57.1)$

$9(75.0) \quad 3(25.0)$

0.130

$0(0) \quad 2(100)$

$7(46.7) \quad 8(53.3) \quad 0.476$

$8(61.5) \quad 5(38.5)$

$8(61.5) \quad 6(38.5) \quad 1.000$

$7(46.7) \quad 7(53.3)$

Serum WBC, $\times 10^{3} / \mathrm{mL}$

$<11$

$9(69.2) \quad 4(30.8) \quad 0.151$

$>11$

$6(40.0) \quad 9(60.0)$

Haemoglobin, g/dL

$>12$

$5(83.3) \quad 1(16.7) \quad 0.173$

$<12$

$10(45.5) \quad 12(54.5)$

Platelets, $\times 1$ 000/L

$<400$

$6(85.7) \quad 1(14.3) \quad 0.084$

$>400$

$9(42.9) \quad 12(57.1)$

Urea, $\mathrm{mmol} / \mathrm{L}$

$<7.2$

$11(52.4) \quad 10(47.6) \quad 1.000$

$>7.2$

$4(57.1) \quad 3(42.9)$

Creatinine, $\mu \mathrm{mol} / \mathrm{L}$

$\begin{array}{lll}<113 & 14(56.0) & 11(44.0) \\ >113 & 1(50.0) & 1(50.0) \\ \begin{array}{l}\text { Missing } \\ \text { Albumin, } \mathrm{g} / \mathrm{L}^{\dagger}\end{array} & 0 & 1(100) \\ <18 & & \\ >18 & 6(42.9) & 8(57.1) \\ \text { Cholesterol, } \mathrm{mmol} / \mathrm{L}^{\dagger} & 9(64.3) & 5(35.7) \\ <12 & & \\ >12 & 8(57.1) & 6(42.9) \\ \text { Missing } & 7(53.8) & 6(46.2) \\ & 0 & 1(100)\end{array}$

0.722

0.449

0.843

${ }^{*}$ Fisher's exact test.

${ }^{* *}$ Adjusted categorical variables with ranges determined by distribution of cohort results rather than normal laboratory values (all patients with abnormal results).

Haematology (full blood count) tests revealed a median (IQR) white blood cell count of $12.7 \times 10^{3} / \mathrm{mL}(8.7-15.2)$, mean (standard deviation (SD)) haemoglobin of $10.8 \mathrm{~g} / \mathrm{dL}$ (2.1), and mean platelet count of $514 \times 10^{3} / \mathrm{mL}$ (no patients had thrombocytopenia). On serum biochemistry tests, median (creatinine was $36.3 \mu \mathrm{mol} / \mathrm{L}$ (IQR 25.1 - 59.2), median (IQR) urea was $4.8 \mathrm{mmol} / \mathrm{L}(2.6$ 7.5), mean (SD) albumin was 19.1 (5.9) g/L, and median (IQR) cholesterol was $11.6 \mathrm{mmol} / \mathrm{L}$ (9.9 - 15.3). All patients had elevated cholesterol and low albumin. 
Table 3. Steroid response by continuous variables

\begin{tabular}{lll}
\hline Variable & $\begin{array}{l}\text { Difference } \\
\text { (SRNS - SSNS patients) }\end{array}$ & $p$-value \\
\hline Age $^{*}$ & -2.2 & 0.084 \\
Systolic blood pressure $^{\dagger}$ & -14.4 & 0.059 \\
Diastolic blood pressure $^{*}$ & -20.0 & 0.004 \\
Serum white blood cells $^{*}$ & 4.7 & 0.084 \\
Haemoglobin $^{\dagger}$ & -2.1 & 0.004 \\
Platelets $^{\dagger}$ & 165.1 & 0.008 \\
Urea $^{*}$ & -3.1 & 0.048 \\
Creatinine $^{*}$ & -16.7 & 0.205 \\
Albumin $^{\dagger}$ & -4.7 & 0.031 \\
Cholesterol $^{*}$ & 1.4 & 0.510 \\
& & \\
SRNS $=$ steroid-resistant nephrotic syndrome; SSNS = steroid-sensitive $^{\text {nephrotic syndrome. }}$ \\
${ }^{*}$ Difference of medians for abnormally distributed variables (Wilcoxon-Mann- \\
Whitney test). \\
${ }^{\dagger}$ Difference of means for normally distributed variables (t-test). \\
\end{tabular}

Response to initial steroid therapy by categorical variables is presented in Table 2. There were no significant differences between the steroid-sensitive and steroid-resistant patients. Analysis of clinical and demographic variables in continuous form was also performed (Table 3). Lower diastolic blood pressure $(p=0.004)$, lower serum haemoglobin $(p=0.003)$, higher platelet counts $(p=0.008)$, lower serum urea $(p=0.05)$, and lower serum albumin $(p=0.03)$ were all significantly higher in patients with steroid resistance.

\section{Discussion}

Steroid responsiveness remains the most important predictive factor for patient outcomes in childhood nephrotic syndrome. ${ }^{[23]}$ Increased cost of healthcare services, inadequate access to longterm dialysis and limited renal transplant capacity all contribute to the increased mortality rate among patients with steroid-resistant disease. ${ }^{[15]}$ Among the 28 patients in our relatively homogenous cohort of black Mozambican children, 13 (46.4\%) had no response to corticosteroid treatment, a relatively high rate of steroid-resistant disease. Conversely, a recent study in Nigeria found the frequency of steroid resistance to be $17.2 \%$ among patients with idiopathic nephrotic syndrome, adding to the growing evidence of geographical or ethnic differences in the steroid response pattern of children with nephrotic syndrome. ${ }^{[4,15,24]}$

Despite this difference in the frequency of steroid resistance, the age profile of patients with steroid-resistant disease reflects that of other cohorts. There was a non-significant association $(p=0.08)$ between young age (when analysed as a continuous variable) and steroid-resistance. Of the patients with steroid resistance in this study, 53.8\% were under the age of five, comparable to the $64 \%$ of steroid-resistant patients under the age of five in the PodoNet Consortium international registry. ${ }^{[5]}$ These findings can likely be explained by the fact that most forms of nephrotic syndrome resulting from genetic mutations, which commonly result in steroid resistance, present early in the life of a child. ${ }^{[5]}$

There was a significant association between normal diastolic blood pressure (when analysed as a continuous variable) and steroid resistance in this cohort, which was an unexpected finding and not consistent with other literature reporting an association between hypertension and steroid resistance. ${ }^{[25]}$ Given the modified approach we had to use for blood pressure classifications, these results should be interpreted with caution.
The lower haemoglobin seen in patients with steroid resistance is likely due to excessive urinary losses of substrates needed for red cell production. These include iron, transferrin, erythropoietin and transcobalamin. ${ }^{[26]}$ We hypothesise that the lower serum albumin seen in patients with steroid-resistant nephrotic syndrome is from the excessive urinary protein loss encountered in these patients. However, the degree of protein loss could not be quantitated, as this study did not assess a urine protein to creatinine ratio but relied on dipstick measurements, which is a semiquantitative measure at best.

When analysed as a continuous variable, higher platelet counts were associated with steroid resistance. A study in Turkey similarly found higher mean platelet counts in a group of patients with steroidresistant FSGS compared with a group with steroid-sensitive disease - suggesting a possible association of elevated platelet counts with disease activity. ${ }^{[27]}$ It has been suggested that the extent of endothelial damage in patients with nephrotic syndrome may be the trigger for thrombocytosis. ${ }^{[28]}$ Another mechanism suggested involves pituitary adenylate cyclase-activating polypeptide (PCAP) - an inhibitor of megakaryopoiesis and platelet aggregability. With urinary loss of PCAP, thrombocytosis and platelet hyperaggregability result. ${ }^{[29]}$ Regardless, our findings of low to normal blood pressure and high platelet count in patients with SRNS compared with SSNS, require further investigation with a larger cohort of patients. One or both of these unique findings could be a prognostic marker for development of steroid-resistant nephrotic syndrome in the future.

Historically, infectious agents have been associated with the development of childhood nephrotic syndrome. The paradigm of a 'tropical nephrotic syndrome', believed to be induced by quartan malaria nephropathy, was introduced in the mid-1970s. ${ }^{[24]}$ However, as access to healthcare services improved in many regions, nephrotic syndrome associated with malaria became increasingly rare in malaria-endemic areas. ${ }^{[30]}$ Mozambique is a malaria-endemic country, but we only had to exclude one nephrotic syndrome patient who tested positive for malaria on admission from our analysis.

However, infectious agents continue to be tied to the development of childhood nephrotic syndrome, with evidence supporting a strong association with hepatitis $B^{[8,31]}$ A study in South Africa showed that the frequency of $H L A-B w 21$, a set of genes associated with susceptibility to the development of membranous nephropathy in response to HBsAg infection, was increased in black patients. ${ }^{[32]}$ There were no patients with a positive HBsAg test in our study; however, only 5 of the 27 patients were tested, which is a limitation of the study. On the other hand, HIV screening was routinely completed in patients admitted for nephrotic syndrome (and one HIV-positive patient was excluded). In our setting, we recommend that all patients with steroid resistance disease undergo testing for hepatitis B and HIV.

Four study patients underwent renal biopsies at outside hospitals. The histological finding for 3 of the patients was FSGS, 2 of whom had steroid-resistant disease. The fourth biopsy showed minimal change disease (MCD) in a patient who had a complete response to steroids. With respect to the histopathology profile of nephrotic syndrome within southern Africa, the occurrence of steroid-sensitive minimal change disease is relatively uncommon. This rise in the incidence of FSGS has been documented in a systematic review of childhood nephrotic syndrome in SSA, including studies from Nigeria, Ghana, the DRC and South Africa, as well as in studies from paediatric patients within the United States. ${ }^{[12-15,24,33-37]}$ In fact, within a South African paediatric cohort, FSGS was the most common lesion among patients with steroid-resistant disease. ${ }^{[38]}$ NPHS2 V260E mutations were found to predict steroid resistance in a cohort of South African children with FSGS and, in a genetic study of European 
patients with African ancestry, mutations in the APOL1 G1 and G2 alleles were seen at a higher frequency in patients with FSGS pathology, suggesting associations between genetic makeup and histopathological diagnosis. ${ }^{[39-41]}$ In light of these associations and the steroid resistance levels at HCM, we recommend expanding capacity for kidney biopsy in those patients who do not have an initial response to corticosteroid therapy.

\section{Study limitations}

There are limitations to our study. It comprised a relatively small cohort of patients which limited power to detect clinical and demographic characteristics associated with steroid resistance. This was also a single-site cohort, and HCM's role as Mozambique's main referral hospital also introduces a potential sampling bias, whereby more complicated cases which were more likely to be steroid-resistant were represented. As this study was retrospective, certain study variables were missing for some included patients, and if not clearly documented in the HCM charts, prior treatment with corticosteroids at outside hospitals before transfer to HCM was missed. Also, owing to limited retrospective data, we had to use a modified approach to blood pressure classifications based on initial inpatient measurements, and as discussed above, our finding of a significant association between normal blood pressure classification and steroid resistance should be interpreted with caution. Furthermore, owing to limited diagnostic capacity in our setting, histopathology and genetic testing were not routinely available, and dipstick urine testing was used instead of the more reliable protein to creatinine ratio to define steroid response.

Despite these limitations, our results add to the evidence base on steroid responsiveness in paediatric nephrotic syndrome within southern Africa and will be a helpful tool to advocate for improved diagnostics and therapeutics in Mozambique.

\section{Conclusion}

In this study, there was relatively low rate of complete response to initial corticosteroid treatment for paediatric nephrotic syndrome compared with other reports from SSA. As early recognition, assessment, and treatment of steroid resistance are important to prevent or delay the onset of CKD and ESRD, HCM needs improved renal laboratory testing, and increased access to kidney biopsy and second-line drugs (cyclophosphamide and calcineurin inhibitors). Future prospective studies should be developed to explore the frequency of relapses after prednisolone treatment, as well as the histopathological diagnosis of children with steroid-resistant disease.

\section{Declarations. None.}

Acknowledgements. We thank the clinical teams in the Department of Paediatrics at Hospital Central de Maputo who cared for the patients included in our study. We also acknowledge the support of the Global Health Program at the UCLA David Geffen School of Medicine.

Author contributions. YM, KF, WCB, EJ and SK contributed to study design. KF and WCB led protocol development and bioethical approvals. YM and KF led data collection. ST and DP led data analysis. KF bore primary responsibility for manuscript drafting. WCB and DP led manuscript revision, with contributions from all authors who approved the final version. EJ and SK were the care providers for the children included in the study.

Funding. None.

Conflicts of interest. None.
1. Spencer JD, Hastings MC, Wyatt RJ, Ault BH. Has the incidence of childhood steroid sensitive nephrotic syndrome changed? Clin Nephrol 2012;78(2):112115. https://doi.org/10.5414/cn107381

2. Ladapo TA, Esezobor CI, Lesi FE. Pediatric kidney diseases in an African country: Prevalence, spectrum and outcome. Saudi J Kidney Dis Transplant 2014;25(5):1110-1116. https://doi.org/10.4103/1319-2442.139976

3. Norris KC, Prabhakar KS, Agodoa L, García-García G. Kidney disease in disadvantaged populations: An unconquered challenge. Clin Nephro 2016;86:S1-2. https://doi.org/10.5414/cnp86s100

4. Olowu WA, Ademola A, Ajite AB, Saad YM. Childhood nephrotic syndrome in tropical Africa: Then and now. Paediatr Int Child Health 2017;37(4):259-268. https://doi.org/10.1080/20469047.2017.1374002

5. Trautmann A, Bodria M, Ozaltin F, et al. Spectrum of steroid-resistant and congenital nephrotic syndrome in children: The PodoNet Registry Cohort. Clin J Am Soc Nephrol 2015;10(4):592-600. https://doi.org/10.2215/cjn.06260614

6. Inguuil E, Tejani A. Racial differences in the incidence and renal outcome of idiopathic focal segmental glomerulosclerosis in children. Pediatr Nephrol 1991;5:393-397. https://doi.org/10.1007/bf01453661

7. Anochie I, Eke F, Okpere A. Childhood nephrotic syndrome: Change in pattern and response to steroids. J Natl Med Assoc 2006;98(12):1977-1981.

8. Abdurrahman M, Fakunle Y, Whittle H. The role of hepatitis B surface antigen in Nigerian children with nephrotic syndrome. Ann Trop Pediatr 1983;3(1):1316. https://doi.org/10.1080/02724936.1983.11748261

9. Adedoyin O, Adesiyun O, Adegboye O, Bello O, Fatoye O. Sickle cell nephropathy in children seen in an African hospital - case report. Niger Postgr Med J 2012;19(2):119-122.

10. Bircan Z, Yilmaz AY, Katar S, Vitrinel A, Yildirim M. Childhood idiopathic nephrotic syndrome in Turkey. Pediatr Int 2002;44:608-611. https://doi. org/10.1046/j.1442-200x.2002.01628.x

11. Niaudet P. Steroid resistant idiopathic nephrotic syndrome in children. In Avner ED, Harmon WE, Niaudet P, editors. Pediatric Nephrology. 5th ed. Philadelphia: Lippincott Williams \& Wilkins; 2003; p. 557.

12. Asinobi A, Ademola A, Okolo C, Yaria J. Trends in the histopathology of childhood nephrotic syndrome in Ibadan Nigeria: Preponderance of idiopathic focal segmental glomerulosclerosis. BMC Nephrology 2015;16(1):1-9. https:// doi.org/10.1186/s12882-015-0208-0

13. Olowu W, Adelusola K, Adefehinti O. Childhood idiopathic steroid resistant nephrotic syndrome in southwestern Nigeria. Saudi J Kidney Dis Transpl 2010;21(5):979-990.

14. Aloni M, Sysleyne L, Ekulu P, Babio F, Ngiyulu R, Gini-Ehungu J. The challenges of caring for children with nephrotic syndrome in a tertiary institution in the Democratic Republic of Congo. Acta Paediatrica 2014;103(8):365-369. https:// doi.org/10.1111/apa.12647

15. Ladapo TA, Esezobor CI, Lesi FE. High steroid sensitivity among children with nephrotic syndrome in southwestern Nigeria. Int J Nephrol 2014;1-6. https:// doi.org/10.1155/2014/350640

16. Asinobi AO, Ademola AD, Ogunkunle OO. Steroid response in primary childhood nephrotic syndrome in a tropical African environment. Niger J Clin Pract 2019;22:790-795. https://doi.org/10.4103/njcp.njcp 20616

17. Seggie J, Davies P, Ninin D, Henry J. Patterns of glomerulonephritis in Zimbabwe: Survey of disease characterised by nephrotic proteinuria. Q J Med 1984;53(209):109-118.

18. Brown K, Abrahams C, Meyers A. The nephrotic syndrome in Malawian blacks. S Afr Med J 1977;52(7):275-278.

19. Coovadia H, Adhikari M, Morel-Maroger L. Clinico-pathological features of the nephrotic syndrome in South African children. Q J Med 1979;48(189):77-91.

20. Downie ML, Gallibois C, Parekh RS, Noone DG. Nephrotic syndrome in infants and children: Pathophysiology and management. Paediatr Int Child Health 2017;37(4):248-258. https://doi.org/10.1080/20469047.2017.1374003

21. Kidney Disease: Improving Global Outcomes (KDIGO) Glomerulonephritis Work Group. KDIGO clinical practice guideline for glomerulonephritis. Kidnet Int Suppl 2012;2(2):1-274. https://doi.org/10.1038/kisup.2012.23

22. National High Blood Pressure Education Working Group on High Blood Pressure in Children and Adolescents. The Fourth Report on the Diagnosis, Evaluation, and Treatment of High Blood Pressure in Children and Adolescents. Pediatrics 2004;114(2 Suppl 4th Report):555-576.

23. International Study of Kidney Disease in Children. Nephrotic syndrome in children: Prediction of histopathology from clinical and laboratory characteristics at time of diagnosis. Kidney Int 1978;13:159-165. https://doi. org/10.1038/ki.1978.23

24. Doe JY, Funk M, Mengel M, Doehring E, Ehrich JHH. Nephrotic syndrome in African children: Lack of evidence for 'tropical nephrotic syndrome'? Nephrol Dial Transplant 2006;21:672-676. https://doi.org/10.1093/ndt/gfi297

25. Shatat IF, Becton LJ, Woroniecki RP. Hypertension in childhood nephrotic syndrome. Front Pediatr. 2019;7:287. https://doi.org/10.3389/fped.2019.00287

26. Iorember F, Aviles D. Anemia in nephrotic syndrome: Approach to evaluation and treatment. Pediatr Nephrol 2017;32:1323-1330. https://doi.org/10.1007/ s00467-016-3555-6

27. Gulleroglu K, Yazar B, Sakalli H, Ozdemir H, Baskin E. Clinical importance of mean platelet volume in children with nephrotic syndrome. Ren Fail 2014;36(5):663-665. https://doi.org/10.3109/0886022x.2014.883931

28. Saigusa T, Kikuchi Y, Yamada M, et al. A case of essential thrombocytosis developing nephrotic syndrome and severe endothelial damage. J Nephrol 2006;19(5):656-659. 
29. Eneman B, Freson K, van den Heuvel L, et al. Pituitary adenylate cyclaseactivating polypeptide deficiency associated with increased platelet count and aggregability in nephrotic syndrome. J Thromb Haemost 2015;13:755-767. https://doi.org/10.1111/jth.12891

30. Olowu WA, Adelusola KA, Adefehinti O, Oyetunji TG. Quartan malariaassociated childhood nephrotic syndrome: Now a rare clinical entity in malaria endemic Nigeria. Nephrol Dial Transplant 2010;25:794-801. https://doi. org/10.1093/ndt/gfp536

31. Abdurrahman $M$. The role of infectious agents in the aetiology and pathogenesis of childhood nephrotic syndrome in Africa. J Infect 1984;8:100-109. https:// doi.org/10.1016/s0163-4453(84)92356-9

32. Adhikari M, Coovadia H, Hammond M. Associations between HLA antigens and nephrotic syndrome in African and Indian children in South Africa. Nephron 1985;41:289-292. https://doi.org/10.1159/000183599

33. Bhimma R, Adhikari M, Asharam K. Steroid-resistant nephrotic syndrome: The influence of race on cyclophosphamide sensitivity. Pediatr Nephrol 2006;21:1847-1853. https://doi.org/10.1007/s00467-006-0276-2

34. Bhimma R, Coovadia H, Adhikari M. Nephrotic syndrome in South African children: Changing perspectives over 20 years. Pediatric Nephrology 1997;11(4):429-434

35. Adhikari M, Bhimma R, Coovadia H. Focal segmental glomerulosclerosis in children from KwaZulu/Natal, South Africa. Clin Nephrol 2001;55:16-24

36. Bonilla-Felix M, Parra C, Dajani T, et al. Changing patterns in the histopathology of idiopathic nephrotic syndrome in children. Kidney Int 1999;55:1885-1890. https://doi.org/10.1046/j.1523-1755.1999.00408.x
37. Uwaezuoke SN, Ndu IK, Mbanefo NR. Prevalence rates of histopathologic subtypes associated with steroid resistance in childhood nephrotic syndrome in Sub-Saharan Africa: A systematic review. Int J Nephrol Renovasc Dis 2019;12:167-176. https://doi.org/10.2147/ijnrd.s207372

38. Bakhiet YM, Mudi A, Khumalo T, Moonsamy G, Levy C. Idiopathic nephrotic syndrome in South African children. Afr Health Sci 2017;17(4):1130-1136. https://doi.org/10.4314/ahs.v17i4.22

39. Govender M, Fabian J, Gottlich E, et al. The podocin V260E mutation predicts steroid resistant nephrotic syndrome in black South African children with focal segmental glomerulosclerosis. Communications Biology 2019;2(1):1-7. https://doi.org/10.1038/s42003-019-0658-1

40. Asharam K, Bhimma R, David VA, et al. NPHS2 V260E is a frequent cause of steroid-resistant nephrotic syndrome in black South African children. Kidney Int Rep 2018;3(6):1354-1362. https://doi.org/10.1016/j.ekir.2018.07.017

41. Gribouval O, Boyer O, Knebelmann B, et al. APOL1 risk genotype in European steroid-resistant nephrotic syndrome and/or focal segmental glomerulosclerosis patients of different African ancestries. Nephrol Dial Transpl 2018;34(11):1-9. https://doi.org/10.1093/ndt/gfy176

Accepted 15 September 2020. 